\title{
CHOICE SYSTEM EN CHILE: DETERMINANTES DEL CAMBIO DE ESCUELA ${ }^{1}$
}

\section{Catalina Canals ${ }^{2}$}

\section{RESUMEN}

Los teóricos del Choice System (Friedman, Chubb, Moe, entre otros) plantearon que la competencia entre establecimientos en mercados escolares y la búsqueda de un ajuste entre las necesidades del estudiante y el establecimiento al cual asiste son dos factores que afectarían el cambio de escuela. Este artículo analiza el efecto de la competencia y del cambio en las características de los establecimientos que pueden afectar el ajuste alumnoescuela, en el cambio de establecimiento en Chile entre 2005 y 2012. Los resultados respaldaron el efecto que tendrían las modificaciones de ciertas características de los establecimientos en el cambio de escuela, hecho pregonado por los teóricos del Choice System, no obstante, no se encontró evidencia de que la competencia entre establecimientos en la comuna afectara dicha tendencia.

Palabras clave: cambio de escuela, competencia, Choice System, mercados educativos.

\section{CHOICE SYSTEM IN CHILE: DETERMINANTS OF SCHOOL SWITCHING}

\section{ABSTRACT}

Choice System theorists (Friedman, Chubb, Moe, among others) argued that competition between schools in school markets and the search for a match between the needs of the student and those of the school, are two factors that could affect school switching. This article analyzes the effect of competition and the changes in schools' characteristics that could affect this school-student fit, in school switching in Chile from 2005 to 2012. The findings support the effect the change of certain school characteristics could have on school switching, proclaimed by the Choice System theorists. However, no evidence was found that competition influences school switching, among schools from the same municipality.

Keywords: School switching, competition, Choice System, school choice, educational markets.

1 Se agradece el financiamiento otorgado por el Proyecto Basal FB0003 del Programa de Investigación Asociativa de Conicyt. Además, se agradece al apoyo de Conicyt, a través del Proyecto Fondecyt Regular No 1120598 , titulado "Estudio de los efectos de la estructuración geográfica sobre la competencia en educación escolar mediante el uso de sistemas de información geográfica y modelamiento basado en agentes".

2 Instituto de Filosofía y Ciencias de la Complejidad, IFICC. Santiago, Chile. Contacto: ccanals@ificc.cl 


\section{Introducción}

Los teóricos del Choice System (en adelante, CS) proponían la existencia de mercados escolares, es decir, por una parte, la libertad de los oferentes para abrir y administrar establecimientos y, por otra, la de las familias para elegir en cuál de ellos educar a sus hijos. Estos sistemas se basarían en la libertad, la competencia y la descentralización (Chubb \& Moe, 1990). La libertad de la demanda educativa estaría expresada en el hecho de que los padres mostrarían su opinión al momento de cambiar a sus hijos de colegio (Friedman \& Friedman, 1982).

La defensa de la libertad, bajo esta perspectiva, tendría efectos positivos en el sistema. La libertad de oferta y de demanda propiciaría un mejor ajuste entre las necesidades del estudiante y la oferta educativa, en la medida en que las familias podían abandonar las escuelas de bajo rendimiento (Elacqua, Martínez, Santos \& Urbina, 2012). Estas "votarían con sus pies y los malos establecimientos verían disminuir sus matrículas y eventualmente serían eliminados" (Mizala y Romaguera, 1998, p. 1). Por su parte, los establecimientos competirían por captar estudiantes, promoviendo la entrega de un servicio de mayor calidad (Cohen-Zada, 2009; Jofré, 1998).

El sistema escolar chileno, instalado en la década del ochenta, profundizó la liberalización de la oferta educativa, entregando subsidios a las escuelas según la asistencia de sus alumnos (Fábrega et al., 2013). Esto en un contexto donde existía libertad formal para elegir cualquier establecimiento. El sistema escolar de Chile es, por tanto, un ejemplo de CS dado que su principal característica es la liberalización de la oferta y la demanda (Chubb \& Moe, 1990).

Siguiendo a Friedman y Friedman (1982) y a Chubb y Moe (1990), en los CS los cambios de escuela serían promovidos por la competencia entre los establecimientos y por el desajuste entre las preferencias familiares y las características de aquellos. Dado lo anterior, la pregunta que pretende responder este estudio es: ¿cuál es el efecto de la competencia en el mercado educativo y de los cambios en las características de los establecimientos en la tendencia al cambio de escuela en Chile? 
En las teorías del CS, el mecanismo fundamental para mejorar la calidad del sistema recae en el hecho de que, ante la insatisfacción de las familias y la presencia de una mejor oferta, estas tenderían a cambiar a sus hijos de escuela. Estudiar si ambos factores impactan o no en esta decisión de los padres permitirá develar si dicho mecanismo existe.

A continuación, se presentará una revisión bibliográfica. Posteriormente, se describirá el modelo de regresión lineal a utilizar, el cual será estimado a partir de Mínimos Cuadrados Ordinarios. Después se detallarán los resultados, los que muestran que las modificaciones en algunas características de los establecimientos afectan el porcentaje de estudiantes que se cambian, pero la competencia local que enfrenta este no lo hace. Por último, se presentan las conclusiones del estudio.

\section{Revisión bibliográfica}

Para examinar los efectos de la competencia y de los cambios en las características de los establecimientos en la movilidad estudiantil en Chile, es relevante referirse de las teorías de CS, a la literatura acerca de los cambios de escuela y a los estudios en torno a la competencia espacial.

\subsection{Las teorías de CS}

Los teóricos del CS proponían sistemas educativos donde hubiese libertad de oferta y de demanda. La primera permitiría abrir y administrar establecimientos; la segunda consistiría en la posibilidad de los padres de elegir el establecimiento de sus hijos, guiados por sus preferencias, y cambiarlos de uno a otro (Friedman, 1950; Friedman, 1995; Friedman \& Friedman, 1982). En Chile, desde 1980 se generaron reformas para potenciar el desarrollo del mercado educacional (Álvarez, 2001; Cornejo, 2006; De la Cruz, 2006; Hsieh \& Urquiola, 2003; Mizala y Romaguera, 1998), creando para ello subsidios estatales que los establecimientos reciben según la asistencia de los estudiantes (Fábrega et al., 2013). Esto hizo del sistema chileno un CS y lo convirtió en un caso relevante a la hora de analizar si los planteamientos de esta perspectiva teórica se evidencian en la realidad nacional. 
Teóricamente, la libertad de oferta y de demanda permitiría un mejor ajuste entre las necesidades del estudiante y las de la escuela, mediante el abandono de aquellas de bajo rendimiento (Elacqua et al., 2012; Mizala y Romaguera, 1998). Esto incentivaría a los establecimientos para entregar un servicio de mayor calidad (Cohen-Zada, 2009; Jofré, 1998) a fin de retener a sus estudiantes y captar otros nuevos. De esta forma, los establecimientos de menor desempeño irían desapareciendo (o perfeccionándose) y la calidad del sistema en su conjunto mejoraría.

Esta promesa se sostenía en los incentivos generados a partir de la competencia entre los colegios, sumado a la búsqueda de un mejor match estudiante-establecimiento en las decisiones familiares. La competencia generaría efectos positivos a través de tres procesos:

- Los establecimientos se verían presionados a mejorar para ser elegidos por los padres.

- Los padres, al ver a otras escuelas prestar un mejor servicio, podrían presionar a los establecimientos donde estudian sus hijos mediante propuestas de mejora (voz en términos de Hirschman, 1970).

- Ante la amenaza de que otros colegios capten a sus alumnos, las escuelas mejorarían su servicio a fin de evitar que sus estudiantes se vayan.

Este estudio se enfocará en el tercero de esos procesos: el cambio de escuela. Desde la perspectiva de los CS, la competencia y el cambio en el ajuste estudiante-escuela serían factores que afectarían dicha decisión. La mejora de la calidad educativa, propuesta por los teóricos, descansaría entonces en los efectos de la competencia en la elección de escuela, la voz y el cambio de establecimiento. De esta forma, si la competencia no interviene, el mecanismo en el cual se basa la mejora de la calidad del sistema planteada por los teóricos no existiría.

\subsection{Cambio de escuela}

Estudios de distintos países han analizado las causas de la elección y el cambio de escuela y las preferencias de los padres que se reflejan 
en estos procesos. En Chile estos tienden a preferir/matricular a sus hijos en establecimientos: con mejores puntajes en pruebas estandarizadas (Canals, 2013; Gallego \& Hernando, 2009; Gómez, Chumacero \& Paredes, 2012), cercanos (Canals, 2013), mixtos (Gallego \& Hernando, 2009), con cierto estatus (Gallego y Sapelli, 2007) y gratuitos, en el caso de los sectores pobres (Córdoba, 2006).

Los establecimientos también exhiben características que afectan el proceso: la dependencia (Dauter \& Fuller, 2011; Joiko, 2012; Loeb \& Valant, 2011; Makovec, Mizala \& Barrera, 2010; Pamies, Carrasco, Beremenyi y Casalta, 2011; Zamora, 2011), su adscripción religiosa (Joiko, 2012), o la calidad; siendo la insatisfacción con el colegio al que asisten sus hijos una de las principales motivaciones para al cambio (Zamora y Moforte, 2013).

Por otro lado, las características de los estudiantes también influyen en el cambio de escuela, entre las variables a destacar están por ejemplo, el ausentismo (Machin, Telhaj \& Wilson, 2006), el nivel de enseñanza o grado (Eadie, Eisner, Miller \& Wolf, 2013; Machin et al., 2006; Zamora, 2011) y el género (Dauter \& Fuller, 2011; Pamies et al., 2011). Adicionalmente, Paredes, Chumacero y Gallegos (2012) muestran que, ante alternativas más económicas, las probabilidades de cambiarse de establecimiento aumentan, evidenciando qué características del mercado influyen en las decisiones.

Todos los factores anteriores identifican rasgos que hacen a los establecimientos o a los estudiantes más propensos al cambio de escuela, pero no precisan qué cambió respecto al momento de elección de escuela que hizo que dicha decisión ya no fuera la preferida. En definitiva, se ha hecho poco énfasis en cómo los cambios en los establecimientos, en sus precios o en el servicio entregado, afectan la movilidad escolar. Este estudio aporta una mirada más dinámica al tema, al considerar que los establecimientos educativos se transforman y que esto podría afectar las decisiones familiares.

Por otro lado, se ha analizado poco el efecto de la competencia en la elección y cambio de escuela. Makovec, Mizala y Barrera (2010) estudian el efecto de la cantidad de oferentes en la probabilidad de 
asistir a un colegio particular subvencionado versus uno público. Sin embargo, no se ha analizado el efecto de la competencia en la tendencia al cambio de establecimiento, lo cual es fundamental para que la pregonada mejora en la calidad del sistema se produzca. Indagar en este tema es relevante en términos de política pública ya que, si la competencia no genera incentivos para mejorar la calidad educativa, habrá que buscar otros modos de generar tal efecto.

Si bien el cambio de escuela puede ser el resultado de la búsqueda de mejores oportunidades -cambios estratégicos- (Loeb \& Valant, 2011), hay traslados que ocurren debido a experiencias negativas (por ejemplo, bajo rendimiento, repitencia, expulsión) llamados cambios reactivos (Loeb \& Valant, 2011).

Por otro lado, los cambios de escuela pueden tener efectos negativos para los estudiantes que se trasladan -debido a dificultades para adaptarse al nuevo establecimiento- (Hanusheck, Kain \& Rivkin, 2001), y efectos disruptivos en las escuelas adonde llegan, al desviar la atención de los profesores hacia la inserción de los estudiantes nuevos (Association of London Goverment, 2005). Considerando las consecuencias de los cambios de escuela, analizar los efectos de la competencia y de las modificaciones en las características de los establecimientos en la movilidad escolar, no solo permite comprobar las predicciones de los teóricos del CS, sino también aportar en la comprensión del fenómeno de la movilidad escolar.

\subsection{Competencia geográfica}

El mercado educativo es geográfico puesto que las familias consideran la distancia a la hora de elegir una escuela para sus hijos (Altonji, Huang \& Taber, 2010; Ansión, Lazarte, Matos, Rodríguez y VegaCenteno, 1998; Bell, 2005; Bernal, 1999; Canals, 2013; Córdoba, 2006; Del Cueto, 2004; Elacqua y Fábrega, 2004; Madero, 2012; Raczynski y Hernández 2011; Van Zantes, 2007). Además, la oferta educativa se distribuye diferenciadamente en los sectores geográficos en, al menos, Santiago (Canals, 2013; Canals, Meneses, Serra y Ministerio de Educación, 2015). 
Para medir la competencia en mercados educativos, se han utilizado diferentes variables: el Índice de Herfindahl e Hirschman (HHI) (Barrow \& Rouse, 2004; Borland \& Howsen, 1993; Dijkgraaf, Van Der Geest, Gradus \& De Jong, 2008), el porcentaje de estudiantes del sector privado -o instrumentos para este- (Cohen-Zada, 2009; Jepsen, 2002; McMillan, 2000), la matrícula por grado (Geller, Sjoquist \& Walker, 2006) y la cuota de mercado (Arum, 1996), entre otros.

Independientemente de la medida, la dificultad estriba en identificar el mercado relevante (Alderighi \& Piga, 2012; Bowblis $\&$ North, 2011), para ello se destacan tres enfoques. El enfoque geopolítico define los límites de los mercados por las fronteras geopolíticas o geográficas. Sin embargo, a veces los consumidores traspasan estos límites para adquirir un servicio y, a veces, se compite en áreas más pequeñas que condados o comunas (Bowblis \& North, 2011). En el enfoque de precios, dos ciudades son de un mismo mercado si los precios del producto varían conjuntamente o difieren en una cantidad menor o igual al costo marginal de transporte (Alderighi, 2012; Morrisey, 1988). Este enfoque no es aplicable en este caso al carecer de información acerca de los costos de los establecimientos. El enfoque de transporte, por otro lado, identifica el área geográfica en la cual cantidades pequeñas del producto son importadas y exportadas (Bowblis \& North, 2011; Chernew, Gowrisankaran \& Fendrick, 2002; Coterill, 1986; Elzinga, 1981; Elzinga \& Hogarty, 1978; Morrisey, Sloan \& Valvona, 1988; Robinson $\&$ Luft, 1985). Esta metodología, requeriría la georreferenciación de estudiantes de todo Chile, información no disponible.

Para este estudio se optó por el enfoque geopolítico, asumiendo como mercado relevante la comuna. Si bien podrían haber sido más pertinentes el enfoque de precios o el de transporte -en tanto hay estudiantes que atraviesan comunas para ir a estudiar (Donoso \& Arias, 2013) - la ausencia de los datos necesarios hace que no sea factible. A pesar de esto, los estudiantes tienden a recorrer distancias pequeñas para ir a la escuela (Canals et al., 2015), por ende, la comuna pareciera ser un área razonable para una primera aproximación al problema. 


\section{Metodología y datos}

Para identificar el efecto de la competencia y de las modificaciones en las características de los establecimientos en la tendencia al cambio de escuela en Chile, se propone un modelo basado en los planteamientos de los teóricos del CS. Desde esta perspectiva, habría dos causas detrás del cambio de escuela: el desajuste de las necesidades de los alumnos con las características del establecimiento i (D) y la competencia en el entorno del establecimiento (CE). Siendo C el porcentaje de estudiantes que se va desde un establecimiento i en el año t (matriculándose en otro en el año siguiente), el modelo podría plantearse de forma lineal del siguiente modo, donde $\beta, \gamma>0$ :

$$
C_{i, t}=\alpha+\beta D_{i, t}+\gamma C E_{i, t}+\varepsilon_{i, t}
$$

Sin embargo, el desajuste entre las necesidades de los alumnos y las características de los establecimientos es difícil de medir, dado que las preferencias varían entre las familias. Por ello, en vez de analizar el efecto de este desajuste en el cambio de escuela, se estudió el efecto de los cambios en las características de los colegios $(\Delta)$, ya que estos modificarían el ajuste establecimiento-estudiante, afectando la movilidad escolar ${ }^{3}$. Particularmente, el cambio en una característica $\mathrm{X}$ en el tiempo t fue medida como:

$$
\Delta x, t=X_{i, t}-X_{i, t-1}
$$

Además, se incorporaron al modelo efectos fijos por año $\left(\tau_{t}\right)$-para controlar tendencias por cada periodo- y efectos fijos

3 Los cambios en las características de los establecimientos pueden generar mayor ajuste estudiante-escuela, menor ajuste, o en caso de indiferencia, igual ajuste. Considerando la heterogeneidad de las preferencias de familias es improbable que ciertos cambios generen indiferencia para todos los estudiantes. De este modo, al cambiar el establecimiento alguna de sus características relevantes el ajuste familia-escuela variará al menos para algunos de sus estudiantes, afectando el porcentaje de estos que se cambia desde el establecimiento. Si el cambio en la característica del establecimiento mejora el ajuste familia-escuela para más familias que para las cuales genera mayor desajuste, el porcentaje de estudiantes que se traslada tendería a disminuir (y viceversa). 
por establecimiento $\left(\boldsymbol{\theta}_{i}\right)$-para controlar por aspectos constantes en el tiempo para los establecimientos que pudieran causar endogeneidad-. Considerando lo anterior, y la disponibilidad de algunas variables de control (VC), el modelo a estimar mediante MCO fue el siguiente:

$$
C_{i, t}=\alpha+\theta_{i}+\tau_{t}+\beta \Delta_{i, t-1}+\gamma C E_{i, t-1}+\mu V C_{i, t}+\varepsilon_{i, t}
$$

Las VC y la CE fueron incluidas de forma rezagada en el modelo -es decir considerando las variables referidas al año anterior- para evitar endogeneidad por simultaneidad.

Desde la perspectiva del CS se esperaban coeficientes $\beta \neq 0$ y $\gamma>0^{4}$. Esto es, que al cambiar las características de los establecimientos, se modificara la tendencia al cambio ${ }^{5}$; y al haber mayor competencia en el mercado donde estaba ubicado el establecimiento, aumentara la tendencia al cambio de escuela.

Para estimar dicho modelo se utilizaron bases de datos del Ministerio de Educación de los años 2004 a 2013, lo que permitió construir una base de datos panel de los establecimientos particulares subvencionados y municipales ${ }^{6}$ de Chile entre los años 2005 y 2012.

Para estimar el porcentaje de estudiantes que se va a otro establecimiento al año siguiente se excluyó a aquellos que estaban en el último grado que ofrecía este y que serían promovidos (dado

4 En aquellos modelos en que para medir competencia se utilicen variables de concentración de mercado, el coeficiente esperado en la regresión estimada será el inverso.

5 Si los cambios en ciertas características mejoran el ajuste familia-escuela para más familias que para las cuales el ajuste disminuye, el porcentaje de estudiantes que se cambia disminuirá $(\beta<0)$, mientras que en el caso contrario, el porcentaje de estudiantes que lo hace aumentará $(\beta>0)$. Encontrar sería indicativo de que las modificaciones de las características no afectan el cambio de escuela, lo que podría deberse a que: a) dichas características son indiferentes para todas las familias, manteniendo invariante la propensión al cambio de todo estudiante; b) el cambio en dichas características mejoró el ajuste para algunos estudiantes, pero disminuyó en otros de modo que se compensen totalmente ambos efectos y el porcentaje de los que se cambian se mantiene. Si bien cuando $\beta=0$, no se puede distinguir el caso a) del b), cuando $\beta \neq 0$ sí se podrá afirmar que el cambio en la característica del establecimiento, modificó el ajuste familia-escuela, afectando el porcentaje de estudiantes que se traslada.

6 No se cuenta con información de precio para establecimientos de otras dependencias. 
que estaban obligados a cambiarse de establecimiento), a los alumnos trasladados a mitad del año y a quienes desertaron del sistema ${ }^{7}$.

Como variables indicativas de los cambios en las características del establecimiento se utilizaron:

- el cambio en el promedio Simce de Matemáticas y Lenguaje estandarizado 8 : Simce $_{t}-$ Simce $_{t-1}$,

- el cambio en el logaritmo del precio: $\ln \left(\right.$ precio $\left._{\mathrm{t}}\right)-\ln \left(\right.$ precio $\left._{\mathrm{t}-1}\right)$,

- el cambio en los sexos admisibles del establecimiento, donde el valor 1 indica que el establecimiento pasó de admitir estudiantes de un solo sexo a ser mixto, y 0 cuando el establecimiento se mantuvo siendo mixto o de un solo sexo.

Se consideraron estas variables dada su disponibilidad y evidencia previa, ya que están dentro de las variables por las cuales los padres tienen preferencias, por lo que su cambio afectaría el ajuste familia-escuela.

Dado el interés en conocer cómo el ajuste entre las preferencias de los padres y las características de los establecimientos influye en el cambio de escuela, se estimaron adicionalmente modelos para escuelas de educación básica en el año 2010. Utilizar un corte transversal implicó omitir los efectos fijos por año y por establecimiento en el modelo, pero en los años 2009 y 2010 el cuestionario Simce de padres y apoderados de $4^{\circ}$ básico incorporó la pregunta respecto de qué tan satisfechos están con la calidad del establecimiento -con valores entre 1 (muy insatisfecho) y 5 (muy satisfecho)-. Esto permitió estimar el promedio de satisfacción con la calidad de este (en el año 2009 y en 2010. De este modo, el modelo para el año 2010 tuvo como variable predictiva el cambio en la satisfacción con la calidad del

7 Se eliminaron a los outliers, definidos como establecimientos con porcentaje de estudiantes que se van del establecimiento estandarizado superior a tres desviaciones estándar. No existían casos con porcentajes estandarizados inferiores a tres desviaciones estándar.

8 En aquellos años donde se rindió Simce en más de un curso se utilizó el promedio del promedio Simce de Matemáticas y Lenguaje estandarizado de cada curso. Para aquellos establecimientos donde no existía el dato del Simce del año anterior, la variable se construyó como Simce -Simce $_{t-2}$. Para los establecimientos sin dato del Simce del año, la variable se construyó como Simce $t_{t-1}$-Simce - $_{-2}$. Esto se consideró oportuno dado que la relevancia de esta variable reside en si las familias observan cambios en este indicador. 
establecimiento correspondiente a . Esta variable no fue incorporada para el análisis panel en tanto no se encontraba en los cuestionarios Simce de todo el periodo.

Por su parte, las variables de competencia fueron:

- la cantidad de establecimientos ${ }^{9}$,

- el $\mathrm{HHI}^{10}$ de la comuna del establecimiento (que indica la concentración del mercado) y,

- la cuota de mercado comunal, correspondiente al porcentaje de estudiantes matriculados en la comuna que corresponden al establecimiento ${ }^{11}$.

Como variables de control se consideró:

- si el establecimiento es municipal o no,

- si el establecimiento está en una zona rural o no y

- el grupo socioeconómico del establecimiento.

Una vez estimados los modelos descritos, se definieron modelos por nivel de enseñanza, considerando como variable dependiente el porcentaje de estudiantes del nivel de enseñanza (básica y media) que se cambió desde el establecimiento. En estos casos los predictores referentes a la competencia fueron estimados considerando solo a aquellos estudiantes y establecimientos del nivel de enseñanza de interés.

La Tabla 1 da cuenta de estadísticos descriptivos de las variables predictoras que fueron utilizadas en los modelos.

9 Si bien muchos oferentes no es sinónimo de competencia, el poder de mercado decrece cuando aumentan los oferentes (Weis, 2002). Esto implicaría que al haber más oferentes tiende a haber mayor competencia.

10 Siendo $m_{i}$ la matrícula de un establecimiento y $m_{z}$ los estudiantes matriculados en la comuna, la cuota de mercado de un establecimiento es $s_{i}=m_{i} / m_{z}$. El índice HHI de la comuna Z, donde hay $\mathrm{N}$ establecimientos, será $H H_{z}=10.000 \cdot \sum_{i=1}^{N} s_{i}^{2}$.

11 Si bien no refiere a la competencia, da cuenta de la posición relativa de un establecimiento en el mercado. Si su cuota de mercado es muy grande, posiblemente se verá menos presionado por la competencia. 
Tabla 1. Estadísticos descriptivos de las variables predictoras

\begin{tabular}{l|c|c|c|c|c}
\hline Variable & Observaciones & Promedio & $\begin{array}{c}\text { Desviación } \\
\text { estándar }\end{array}$ & Mínimo & Máximo \\
\hline Cambio en sexos admisibles & 44.982 & 0,0027 & 0,0518 & 0 & 1 \\
\hline Cambio en precio & 44.982 & 0,0167 & 0,904 & $-10,9478$ & 10,9478 \\
\hline Cambio en Simce & 44.982 & 0,0059 & 0,6374 & $-3,9975$ & 4,6239 \\
\hline Valor absoluto de cambio en precio & 44.982 & 0,1412 & 0,8931 & 0 & 10,9478 \\
\hline Valor absoluto de cambio en Simce & 44.982 & 0,4824 & 0,4166 & 0,0000005 & 4,6239 \\
\hline HHI comunal & 44.982 & 786,2818 & 875,5712 & 120,9882 & 10.000 \\
\hline Cuota de mercado comunal & 44.982 & 0,0519 & 0,0888 & 0,0001 & 1 \\
\hline $\begin{array}{l}\text { Cantidad de establecimientos en la } \\
\text { comuna }\end{array}$ & 44.982 & 50,6023 & 33,7294 & 1 & 133 \\
\hline $\begin{array}{l}\text { Cantidad de establecimientos de ed. } \\
\text { básica en la comuna }\end{array}$ & 43.208 & 46,4539 & 30,9279 & 1 & 127 \\
\hline HHI ed. básica comunal & 43.208 & 830,9752 & 916,2483 & 119,046 & 10.000 \\
\hline Cuota de mercado ed. básica comunal & 43.208 & 0,0573 & 0,0942 & 0,0001 & 1 \\
\hline $\begin{array}{l}\text { Cantidad de establecimientos de ed. } \\
\text { media en la comuna }\end{array}$ & 13.167 & 22,9458 & 19,277 & 1 & 75 \\
\hline HHI ed. media comunal & 13.167 & $1.817,711$ & $2.131,951$ & 206,1433 & 10.000 \\
\hline Cuota de mercado ed. media comunal & 13.167 & 0,1256 & 0,2113 & 0,0001 & 1 \\
\hline
\end{tabular}

Fuente: Elaboración propia.

Dado que la información disponible es censal para los establecimientos de la población, la muestra incluyó a aquellos que disponían de información utilizable para realizar el análisis. Dado que no era una muestra probabilística y que el bootstrap no paramétrico no se ve afectado por los datos perdidos (Efron, 1992), los errores estándar de los coeficientes de los modelos fueron estimados mediante este método. La muestra incluyó a 6.961 de los 9.816 establecimientos municipales y particulares subvencionados de niños y jóvenes del país del periodo ${ }^{12}$.

\section{Resultados}

Previamente a estimar los modelos, parece relevante describir las relaciones bivariadas entre los predictores y el porcentaje de estudiantes que se cambia de establecimiento. La Figura 1 muestra

12 Dado que la muestra correspondió a la mayoría de la población, analizarla sin necesidad de hacer inferencia ayuda a comprender cómo ocurre el fenómeno en la mayoría de los establecimientos del país. 
la relación entre el porcentaje de estudiantes que se cambia de los establecimientos e indicadores de competencia. Las Figuras 1.A y 1.B muestran relaciones negativas entre $\mathrm{HHI}$ y la cuota de mercado, con el porcentaje de estudiantes que se cambia desde un establecimiento; esto sugeriría que el cambio de escuela suele ser menor en los mercados concentrados y para los establecimientos con posiciones más privilegiadas en su mercado; coincidiendo con lo esperado por los teóricos de CS. La Figura 1C muestra una relación menos clara entre la cantidad de establecimientos en la comuna y la tendencia al cambio, habiendo una correlación muy baja entre ambas variables.

Figura 1. Relación entre el Porcentaje de estudiantes que se cambian desde el establecimientos e indicadores de mercado

A.

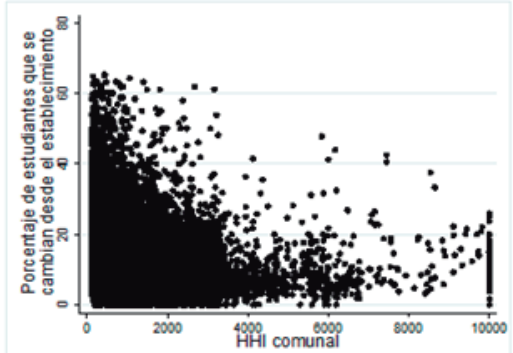

$R$ de Pearson $=-0.1692$

B.

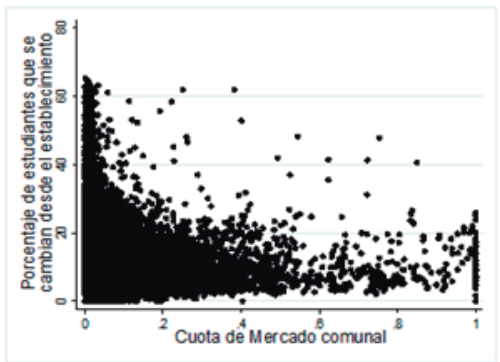

$R$ de Pearson $=-0.1594$

C.

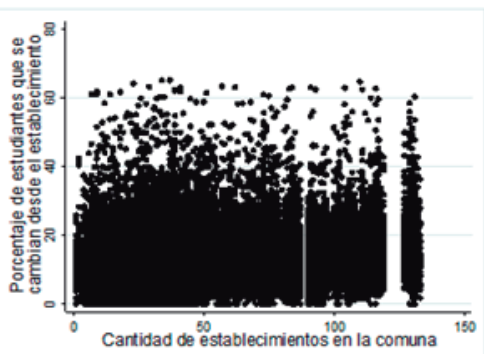

$R$ de Pearson=-0,1878 
La Figura 2 presenta las relaciones entre las variables relativas a las modificaciones en características de los establecimientos y el porcentaje de estudiantes que se cambia de estos. Modificaciones en el precio, en el Simce y en los sexos admisibles no parecieran relacionarse linealmente con el porcentaje de estudiantes que se traslada de establecimiento (Figura 2.A, 2.C y 2.E). En las Figuras 2.B y 2.D, pareciera observarse una relación negativa entre el valor absoluto del cambio del Simce y el valor absoluto del cambio del precio con el cambio de escuela, pero las correlaciones entre las variables son muy bajas y en el caso del precio esta es positiva. A fin de comprender mejor las relaciones entre estas variables y el cambio de escuela, adicionalmente se estimaron modelos utilizando el valor absoluto del cambio en precio y en Simce como variables predictivas.

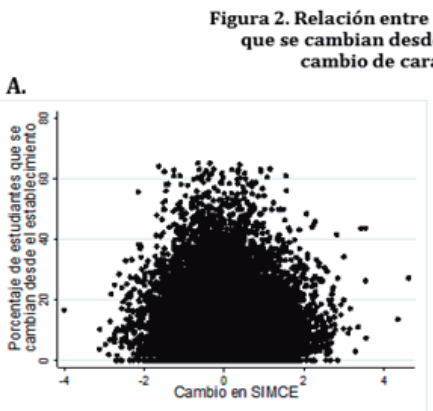

$R$ de Pearson=- 0.0071

C.

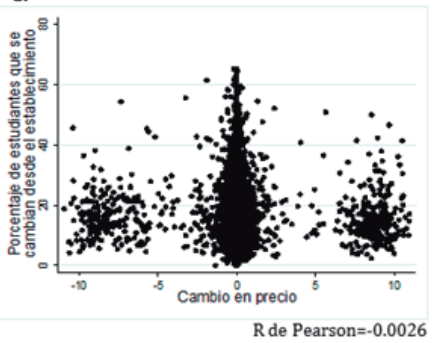

D.

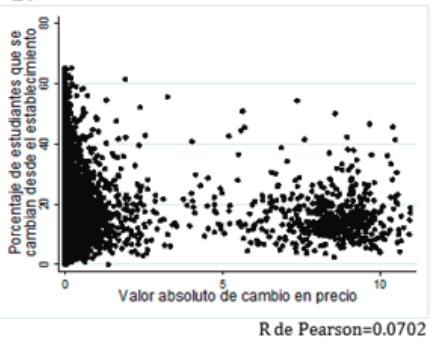

E.

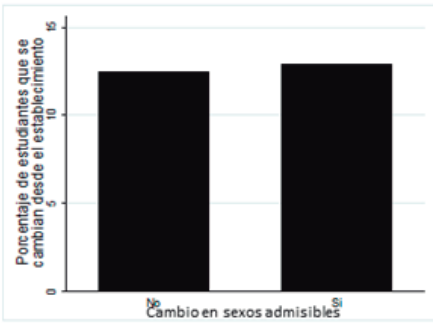




\subsection{El cambio en las características de los establecimientos y el cambio de establecimiento}

Los resultados de los modelos están sintetizados en las Tablas 2 y 3. Los predictores de los modelos de la Tabla 3 que corresponden a cambios en las características de los establecimientos son: cambio en sexos admisibles, en precio y en Simce, mientras que los de los modelos de la Tabla 2 son: cambio en sexos admisibles, valor absoluto del cambio en precio y en Simce. Se puede notar que los modelos donde los predictores corresponden al valor absoluto de los cambios (Tabla 3), en vez del cambio en Simce y el cambio en precio (Tabla 2) presentan un ajuste igual o superior, pareciendo estos modelos más pertinentes.

Los modelos presentados en ambas tablas, permiten afirmar con 95\% de confianza, que los cambios en los sexos admisibles - pasar de ser un establecimiento de un solo sexo a uno mixto- no genera un efecto distinto de cero en el porcentaje de estudiantes que se cambian. Aunque los coeficientes estimados no siempre tienen el mismo signo, la magnitud es siempre inferior a 1 , sugiriendo que el efecto de la variable es escaso o nulo en la tendencia al cambio de escuela ${ }^{13}$.

13 Esto podría deberse a la escasa cantidad de establecimientos que cambiaron los sexos admisibles durante el periodo de estudio (109). Dado lo anterior, de forma adicional, se estimaron los modelos restringiendo la muestra a estos 109 establecimientos, obteniendo coeficientes de magnitudes inferiores al 1 . Esto evidencia que, incluso en la muestra de establecimientos que cambia sus sexos admisibles en el periodo, el impacto de esto en el traslado de sus estudiantes ha sido muy menor. 


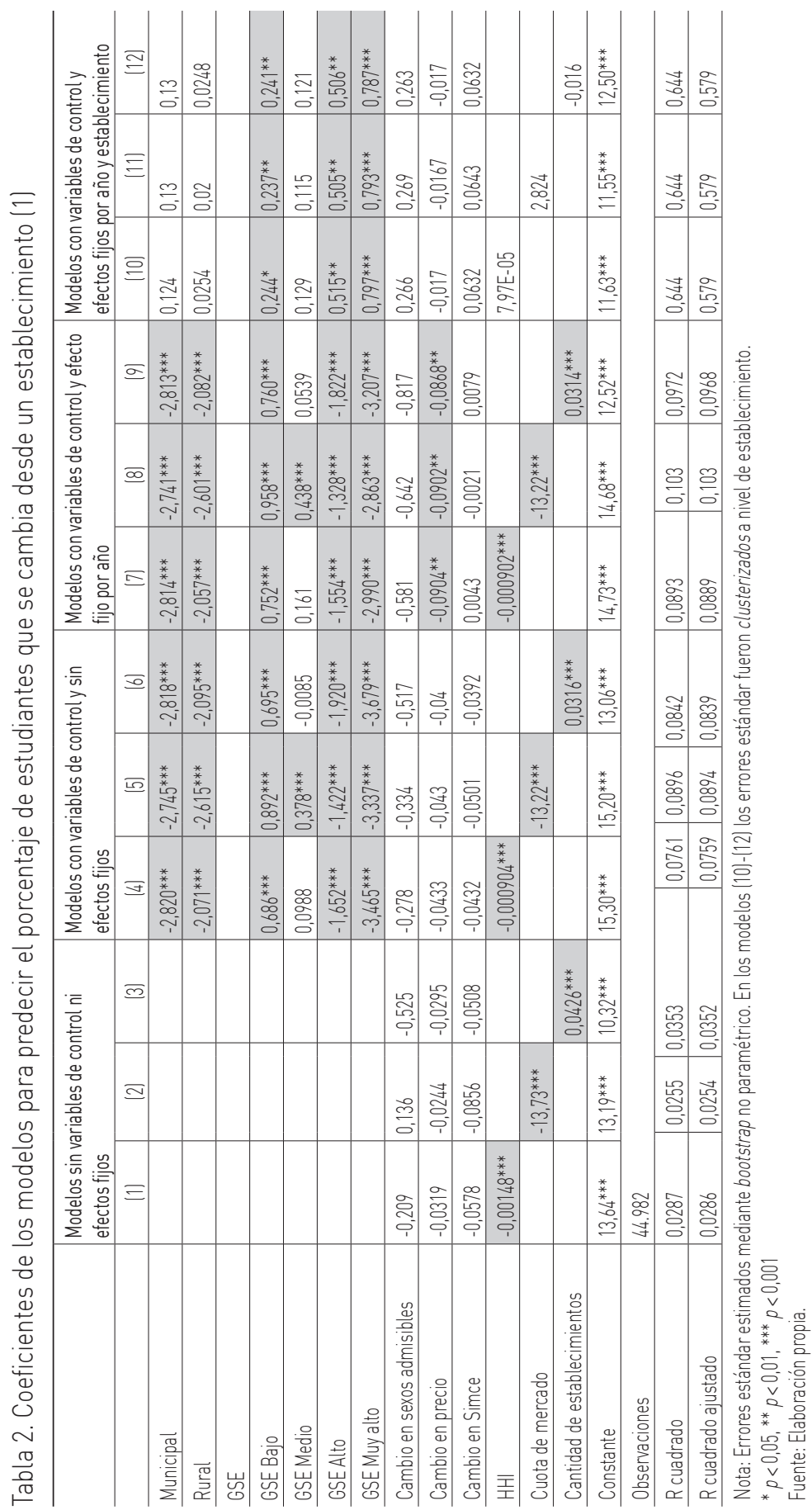




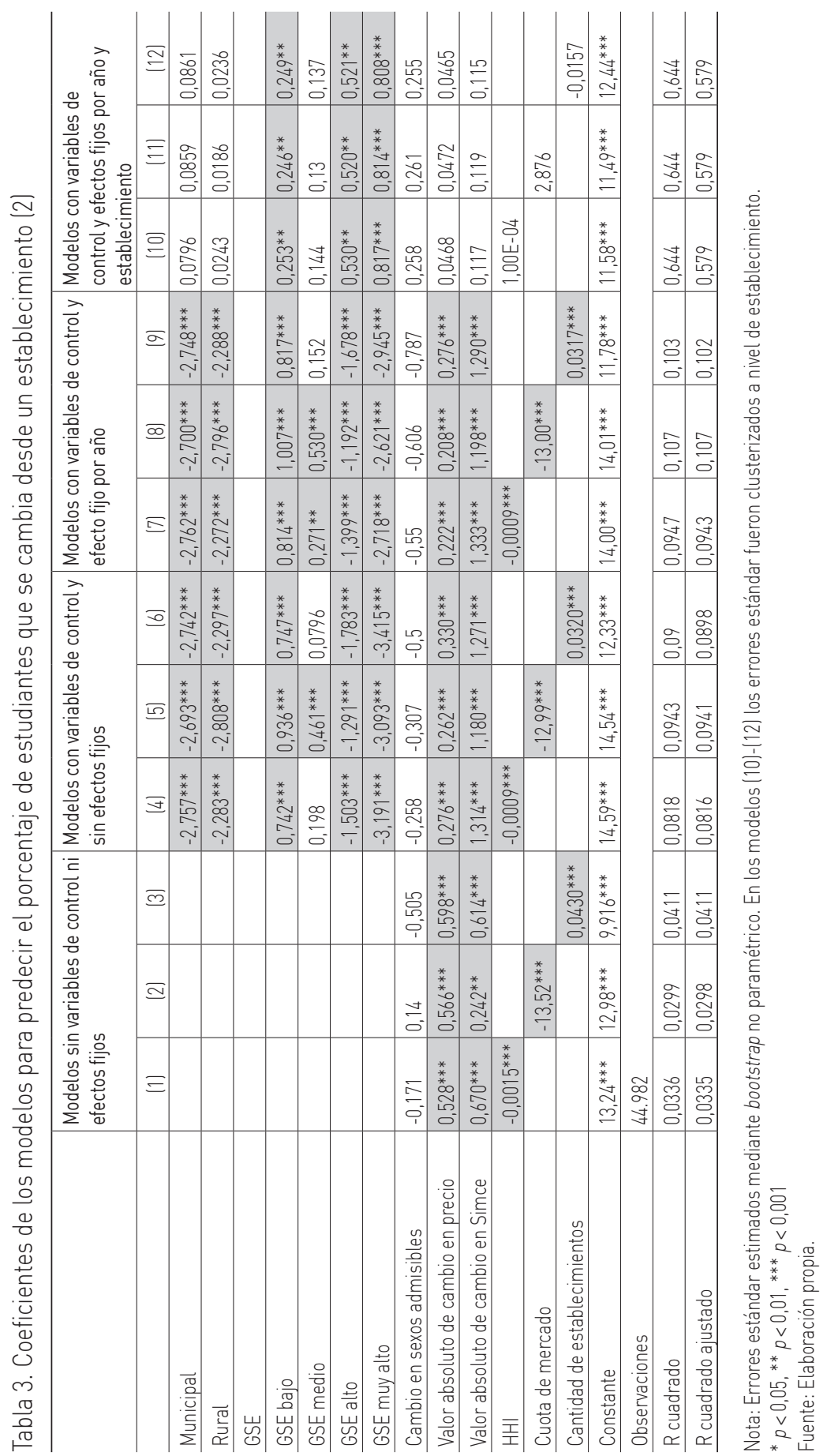


Por otro lado, ninguno de los modelos (Tabla 2) permite afirmar que cambios en el Simce generen un efecto distinto de cero (con 95\% de confianza) en los estudiantes que se cambian. Sin embargo, en los modelos (1)-(9) de la Tabla 3 (modelos sin efecto fijo por establecimiento), se observa que, con 95\% de confianza, el efecto del valor absoluto del cambio en el Simce en el porcentaje de estudiantes que se cambian sí es distinto de cero. Los modelos predecirían que un establecimiento con el máximo del valor absoluto del cambio en Simce (4,6 aprox.) tendría entre 5,5\% y $6 \%$ más estudiantes que se cambian desde el establecimiento ${ }^{14}$.

En cuanto al cambio en el precio del establecimiento se puede notar que, solo en los modelos con variables de control y efecto fijo por año de la Tabla 2 (modelos (7)-(9)), se puede afirmar que el efecto sea, con 95\% de confianza, distinto de cero. A pesar de ello, los coeficientes estimados siempre son negativos indicando que, al aumentar el precio, la tendencia al cambio en los establecimientos de la muestra disminuye. En los modelos que utilizan como predictor el valor absoluto del cambio en el precio (Tabla 3) sin efecto fijo por establecimiento (modelos (1)-(9)) se observa que, con 95\% de confianza, el efecto de esta variable en los estudiantes que se cambian es distinto de cero. A partir de estos modelos, un establecimiento con el máximo del valor absoluto del cambio del precio (11 aproximadamente) tendría un porcentaje de estudiantes que se va entre $2,2 \%$ y $6,6 \%$ mayor que uno que no cambia su precio.

Contrariamente a los modelos (1)-(9) de la Tabla 3, los modelos (10)-(12), que incluyen efectos fijos por establecimiento, no permiten afirmar que los efectos de los cambios en el valor absoluto del cambio en Simce y precio sean distintos de cero en la población; aun cuando los signos de los coeficientes son consistentes con los modelos previos. Esto podría deberse a la baja varianza del valor absoluto del cambio en Simce y precio de un mismo establecimiento de la muestra entre 2005 y $2012^{15}$.

14 Esto evidenciaría que la relación negativa que parecía observarse en la Figura 1.B se revierte al incluir variables de control.

15 En promedio la desviación estándar del valor absoluto del cambio en el precio y Simce de un establecimiento de la muestra es 0,2 y 0,3 , respectivamente. Además, la mayoría de la muestra (4.870 establecimientos) no cambia nunca su precio. 
Dado esto, se estimaron los modelos con variables de control, efecto fijo por año y por establecimiento, con la muestra de establecimientos que varió su precio en el periodo, y la de aquellos con valor absoluto de cambio en Simce superior a 0,27 (correspondiente a la mediana). Para aquellos establecimientos cuyo precio se modificó, el mayor valor absoluto del cambio en precio se asocia con una mayor tendencia al cambio, aun cuando, con 95\% de confianza, esto no se puede extrapolar a la población. Para los establecimientos con variaciones absolutas del Simce superiores a la mediana, con 95\% de confianza, estas generan un efecto distinto de cero en la tendencia al cambio de establecimiento, la que aumenta al haber un mayor valor absoluto del cambio en el Simce.

Los resultados anteriores no permiten afirmar que, a mayor valor absoluto del cambio en precio, mayor sea la tendencia al cambio en los establecimientos de la población. Aun así, esto sí sucede en la muestra y, por ende, en la mayoría de los establecimientos. Además, los resultados sugieren que aumentar el valor absoluto del cambio en el Simce de los establecimientos de la población se asociaría a un mayor porcentaje de estudiantes que se cambian.

Desde las perspectivas económicas más clásicas, se esperaría que las familias se cambiaran más solo ante alzas de precios. Sin embargo, en la muestra los aumentos y también las disminuciones de precio propiciarían el cambio de escuela. Posiblemente existe un doble efecto: familias con presupuestos restringidos aumentan su tendencia al cambio ante alzas de precios, mientras que familias con mayor presupuesto lo harían ante las disminuciones de precio. Bajar el precio podría fomentar el cambio al permitir la entrada al establecimiento de estudiantes de menor nivel socioeconómico. Esto podría generar un menor ajuste estudiante-escuela para algunos padres que prefieran que sus hijos interactúen con niños de su mismo nivel socioeconómico (Elacqua, Santos, Urbina y Martínez, 2011) y no lo hagan con estudiantes de menor nivel socioeconómico, al asociarlos a la imagen de flaite (Canales, Bellei y Orellana, 2016, en prensa).

Los efectos del aumento del valor absoluto del cambio en el Simce en el cambio de escuela también parecieran contradecir 
a la teoría ya que, según esta, los padres cambiarían a sus hijos de establecimientos de mala calidad (Mizala y Romaguera, 1998). Sin embargo, el Simce no necesariamente representa el ideal educativo de los padres (Ortiz, 2012), pudiendo existir dos tendencias que expliquen este efecto. Las disminuciones del Simce podrían generar en algunos los padres una menor valoración del establecimiento, al asociar Simce a calidad, aumentando el cambio de escuela ${ }^{16}$. Por otro lado, mayor Simce podría potenciar el cambio de escuela, ya con ello el establecimiento podría descuidar otras características valoradas por otros padres.

Los modelos de la Tabla 4 permiten analizar si existen diferencias entre la educación básica y la educación media. Los resultados son consistentes con los resultados generales, en tanto mayor valor absoluto del cambio en precio y Simce aumenta el porcentaje de estudiantes que se cambia. Sin embargo, las magnitudes de los efectos difieren: mientras en educación básica el efecto de una variación del precio en la tendencia al cambio es mayor que en enseñanza media, el efecto de una variación del Simce durante la enseñanza media es mayor que en la enseñanza básica. Estos resultados sugieren que los resultados de los test estandarizados son más relevantes para las familias al aproximarse el egreso de la educación escolar, mientras que los precios pierden importancia.

16 Esto es consistente con que los resultados en los test estandarizados inciden en la elección de escuela (Canals, 2013; Gallego \& Hernando, 2009; Gómez et al., 2012). 
Tabla 4. Coeficientes de los modelos para predecir el porcentaje de estudiantes que se cambia desde un establecimiento según nivel de enseñanza

\begin{tabular}{|c|c|c|c|c|c|c|}
\hline & \multicolumn{3}{|c|}{$\begin{array}{c}\text { Variable dependiente: porcentaje } \\
\text { de estudiantes que se cambia } \\
\text { desde un establecimiento en } \\
\text { educación básica }\end{array}$} & \multicolumn{3}{|c|}{$\begin{array}{c}\text { Variable dependiente: porcentaje } \\
\text { de estudiantes que se cambia } \\
\text { desde un establecimiento en } \\
\text { educación media }\end{array}$} \\
\hline & (1) & (2) & (3) & (4) & (5) & (6) \\
\hline Municipal & $-2,556 * * *$ & $-2,660 * *$ & $-2,665 * *$ & $-0,957 * * *$ & $-1,923^{* * *}$ & $-1,542 * * *$ \\
\hline Rural & $-3,123^{* * *}$ & $-2,615 * * *$ & $-2,562^{* * *}$ & 0,145 & 0,268 & $0,574^{* *}$ \\
\hline \multicolumn{7}{|l|}{ GSE } \\
\hline GSE bajo & $0,899 * * *$ & $0,678 * *$ & $0,670 * * *$ & $0,635 * *$ & $0,657^{* *}$ & $0,647^{* *}$ \\
\hline GSE medio & $0,258 *$ & $-0,141$ & $-0,0414$ & $-0,505 *$ & $-0,384$ & $-0,424$ \\
\hline GSE alto & $-1,446^{* * *}$ & $-1,888 * * *$ & $-1,647^{* * *}$ & $-3,078^{* * *}$ & $-2,947 * *$ & $-2,952 * * *$ \\
\hline GSE muy alto & $-2,997 * * *$ & $-3,373^{* * *}$ & $-3,067 * *$ & $-3,058^{* * *}$ & $-3,007 * *$ & $-3,098 * * *$ \\
\hline $\begin{array}{l}\text { Cambio en sexos } \\
\text { admisibles }\end{array}$ & $-0,934$ & $-1,001$ & $-0,85$ & 0,0809 & 0,205 & 0,135 \\
\hline $\begin{array}{l}\text { Valor absoluto de } \\
\text { cambio en precio }\end{array}$ & $0,240^{* * *}$ & $0,316^{* * *}$ & $0,257 * *$ & 0,0814 & $0,137^{* * *}$ & $0,0871^{*}$ \\
\hline $\begin{array}{l}\text { Valor absoluto de } \\
\text { cambio en Simce }\end{array}$ & $0,983 * * *$ & $1,084^{* * *}$ & $1,137^{* * *}$ & $1,806 * * *$ & $1,975 * *$ & $1,964^{* * *}$ \\
\hline $\mathrm{HHI}$ & $-13,56^{* * *}$ & & & $-8,117 * *$ & & \\
\hline Cuota de mercado & & $0,0353 * * *$ & & & $0,0609 * * *$ & \\
\hline Cantidad de colegios & & & $-0,000955^{\cdots \cdots}$ & & & $-0,000585 \cdots$ \\
\hline Constante & $14,44^{* * *}$ & $12,09 * * *$ & $14,42^{* * *}$ & $14,26 * * *$ & $12,03^{* * *}$ & $14,41 * * *$ \\
\hline Observaciones & \multicolumn{3}{|l|}{43.208} & \multicolumn{3}{|l|}{13.167} \\
\hline R cuadrado & 0,109 & 0,101 & 0,0941 & 0,0934 & 0,0745 & 0,0747 \\
\hline R cuadrado ajustado & 0,108 & 0,1 & 0,0937 & 0,0922 & 0,0733 & 0,0735 \\
\hline
\end{tabular}

Nota: Errores estándar estimados mediante bootstrap. Modelos estimados con efectos fijos por año. ${ }^{*} p<0,05,{ }^{* *} p<0,01,{ }^{* * *} p<0,001$

Fuente: Elaboración propia.

Considerando que hay padres con nociones de calidad distinta de la de los resultados Simce (Ortiz, 2012), se estimaron modelos con los establecimientos de educación básica en el año 2010 que incluyeron como variable predictiva el cambio en la satisfacción con la calidad del establecimiento. Los resultados de estos (Tabla 5) son consistentes con los de los modelos (1)-(9) de la Tabla 3, en tanto que al aumentar el valor absoluto del cambio en precio y Simce se incrementa el porcentaje de estudiantes que se cambia. Adicionalmente, al aumentar la satisfacción de los padres con la calidad del establecimiento el porcentaje de estudiantes que se cambia, disminuye (y viceversa). 
De este modo, los resultados de los modelos estimados avalarían las teorías de CS, en la medida en que al cambiar algunas de las características de los establecimientos, la tendencia a cambiarse de establecimiento se modifica. Esto sugeriría que, los cambios en las características de los establecimientos, dadas las preferencias de los padres, afectan el ajuste estudiante-escuela. Luego, aquellos padres con mayor desajuste harían más probable su cambio de establecimiento.

Tabla 5. Coeficientes de los modelos para predecir el porcentaje de estudiantes de educación básica que se cambia desde un establecimiento en el año 2010

\begin{tabular}{l|c|c|c}
\hline & $(1)$ & $(2)$ & $(3)$ \\
\hline Municipal & $-2,027^{* * *}$ & $-2,110^{* * *}$ & $-2,088^{* * *}$ \\
\hline Rural & $-2,863^{* * *}$ & $-2,371^{* * *}$ & $-2,296^{* * *}$ \\
\hline GSE & & & \\
\hline GSE bajo & $1,013^{* * *}$ & $0,813^{* * *}$ & $0,795^{* * *}$ \\
\hline GSE medio & 0,434 & 0,0333 & 0,135 \\
\hline GSE alto & $-0,728^{*}$ & $-1,227 * * *$ & $-1,053^{* * *}$ \\
\hline GSE muy alto & 0,537 & 0,357 & 0,448 \\
\hline Cambio en sexos admisibles & 1,539 & 0,882 & 1,667 \\
\hline Valor absoluto de cambio en precio & $0,519^{* * *}$ & $0,585^{* * *}$ & $0,557 * *$ \\
\hline Valor absoluto de cambio en Simce & $0,466^{*}$ & $0,536^{*}$ & $0,601 * *$ \\
\hline Cambio en satisfacción con calidad & $-1,417 * *$ & $-1,388^{* * *}$ & $-1,420^{* * *}$ \\
\hline HHI & $-12,30^{* * *}$ & & \\
\hline Cuota de mercado & & $0,0353^{* * *}$ & \\
\hline Cantidad de establecimientos & & & $-0,00103^{* * *}$ \\
\hline Constante & $13,46^{* * *}$ & $11,22^{* * *}$ & $13,61^{* * *}$ \\
\hline Observaciones & & & 5.235 \\
\hline R cuadrado & 0,102 & 0,0988 & 0,0927 \\
\hline R cuadrado ajustado & 0,1 & 0,0969 & 0,0907 \\
\hline
\end{tabular}

Nota: Errores estándar estimados mediante bootstrap.

${ }^{*} p<0,05,{ }^{* *} p<0,01,{ }^{* * *} p<0,001$

Fuente: Elaboración propia.

\subsection{La competencia en la comuna de los establecimientos y el cambio de establecimiento}

Respecto de la competencia, los modelos (1)-(9) de las Tablas 2 y 3 indicarían que, con 99,9\% de confianza el HHI, la cuota de mercado y la cantidad de establecimientos en la comuna impactan en el porcentaje de estudiantes que se cambian de establecimiento, pero en magnitudes pequeñas. Aumentar en 10.000 el HHI (pasar desde 
la total a nula competencia) incrementaría entre un 9\% y un 15\% la cantidad de estudiantes que se cambian, al hacerlo en $100 \%$ la cuota de mercado se acrecentaría entre un 13\% y 14\% y al aumentar en 100 los establecimientos en la comuna la cantidad de estudiantes que se traslada se crecería entre un $3 \%$ y un $4,3 \%$.

Sin embargo, al incluir en los modelos un efecto fijo por establecimiento, los efectos de estas variables ya no se pueden afirmar que sean distintos de cero en la población y, además, cambian de signo. Si bien esto podría deberse a que, los establecimientos tienen baja varianza en estas variables durante el periodo ${ }^{17}$, al estimar los modelos con variables de control y efecto fijo por establecimiento, considerando solo aquellos con desviaciones estándar en las variables por sobre la mediana, los coeficientes estimados mantienen los signos de los modelos (10)-(12) de las Tablas 2 y 3 (sin ser significativos, con $95 \%$ de confianza). De este modo, no se puede afirmar que en la muestra ni en la población una mayor competencia en la comuna de un establecimiento (menor HHI y más establecimientos) y una posición menos privilegiada (menor cuota de mercado) genere mayor tendencia al cambio de escuela.

Considerando estos resultados, por un lado, las estimaciones sugieren que los establecimientos con menor HHI, más oferentes y menor cuota de mercado tienen un mayor porcentaje de estudiantes que se cambian (como muestran los modelos (1)-(9) de las Tablas 2 y 3 ). Por otro lado, el hecho de que al incluir el efecto fijo estos resultados se modifiquen sugiere que un mismo establecimiento no aumenta su porcentaje de estudiantes que se cambia cuando su HHI y cuota de mercado disminuyen y sus competidores aumentan. Esto indicaría que las familias no responden a los cambios en el mercado local de un establecimiento, pero sí hay más propensión al cambio desde aquellos establecimientos que durante el periodo tienen mayor competencia.

El hecho de que los cambios en el mercado local de un establecimiento no afecten la propensión al cambio podría deberse

17 La desviación estándar de la cantidad de colegios en la comuna, del HHI y de la cuota de mercado de un mismo establecimiento es en promedio $1,58,44$, y 0,005, respectivamente. 
a una suerte de inercia, al haber una baja conducta potencial de cambio escolar motivado por la insatisfacción con el establecimiento (Corvalán y Román, 2012). Las familias podrían ser poco propensas al cambio por los costos de adaptación que puede tener para el estudiante (Hanusheck et al., 2001), porque su hijo no quiere cambiarse (Corvalán y Román, 2012), u otros motivos. De este modo, la aparición de nuevas alternativas en la zona (o de más competencia), considerando que la educación es un bien de calidad oculta, no logra incentivar el cambio.

Por su parte, el que establecimientos de sectores con más oferentes y más competencia tengan mayor tendencia al cambio (como muestran los modelos (1)-(9)) pareciera deberse a problemas de endogeneidad, en tanto la competencia se asociaba a aspectos constantes en los establecimientos que sí influyen en el cambio de escuela. Estos aspectos podrían ser otras características de los establecimientos asociados a la competencia (por ejemplo, conectividad y acceso a servicios públicos, culturales y deportivos en la zona), o podría ser la competencia pasada del establecimiento ${ }^{18}$. Por ejemplo, podría suceder que, en sectores con más oferentes, en el momento de elegir una escuela, a los padres les resulte más difícil identificar aquel que satisface mejor sus necesidades, ya que la existencia de muchas alternativas puede generar desorientación (Usón, 1993). Dado esto, con posterioridad se podrían producir más cambios en dichas zonas, en tanto la opción elegida no es la de mejor ajuste estudiante-escuela.

Aun así, dado que los modelos con efecto fijo muestran que los padres no son sensibles a los cambios en la competencia de un mismo establecimiento, la transición de un mercado comunal hacia uno más competitivo no incentivaría a los padres a cambiar a sus hijos de escuela. Al no hacerlo, la competencia no incentivaría a los establecimientos a mejorar. Esto se contrapondría al planteamiento de la teoría de los CS de que mayor competencia generaría mayor cambio de escuela.

18 La cual, dada la poca varianza de la competencia de un mismo establecimiento, se relaciona con la competencia actual. 


\subsection{Comentario metodológico}

Una limitación de los modelos estimados es que aun cuando el efecto fijo por establecimiento permite controlar problemas de endogeneidad por variables constantes en el tiempo para los establecimientos, se sabe que los cambios de escuela se ven afectados por factores que no son constantes en el tiempo. Distintos cambios del establecimiento (por ejemplo, cambios en la política de expulsión de estudiantes, en la dirección del establecimiento, en el proyecto educativo), y distintos cambios en los estudiantes (por ejemplo, variaciones en el porcentaje de estudiantes cuyos padres pierden el empleo, aumento de las distancias promedio estudiante-escuela) pueden afectar el porcentaje de estudiantes que se va de una escuela. Estos factores, si inciden en la propensión al cambio, estarían en el error de los modelos estimados. En la medida en que estas variables omitidas se correlacionen con las variables incluidas, tendremos problemas de endogeneidad y, por ende, sesgo en las estimaciones.

Considerando que las variables incluidas en los modelos refieren a cambios en las características de los establecimientos y a la competencia, parece improbable que las variables omitidas referentes a los cambios en las características de los estudiantes se relacionen con estas y, por tanto, generen problemas de endogeneidad. Las variables omitidas referidas a cambios en características del establecimiento sí podrían estar relacionadas con los predictores de los modelos descritos $y$, por ende, generar problemas de endogeneidad. Por ejemplo, un cambio de un director podría asociarse a una nueva orientación de un establecimiento que tenga como resultado un mayor o menor Simce (y por ende un mayor valor absoluto del cambio en el Simce); en dicho caso, el efecto estimado del cambio del Simce en el modelo presentado podría estar sobreestimado, en tanto la variable omitida (cambio del director) se correlaciona positivamente con la variable incluida (cambio en el valor absoluto del Simce).

Similarmente, cambios en otras características de los establecimientos omitidas en los modelos, podrían asociarse con mayor valor absoluto del cambio del Simce o del precio, produciendo sobreestimación del efecto de tales variables en la tendencia al cambio. 
Aun cuando puede que se estén sobreestimando los efectos del valor absoluto del cambio en el Simce y en el precio, es importante notar que en los modelos para predecir el porcentaje de estudiantes de educación básica que se cambia en el año 2010 (Tabla 5), se incorporó la variable predictiva cambio en la satisfacción con la calidad del establecimiento, la cual debiera asociarse con las variables omitidas que generaban problemas de endogeneidad en los modelos longitudinales. De este modo, los modelos para el año 2010 no tendrían tal problema de omisión de variables relevantes y aun así muestran que el valor absoluto de los cambios de precios y Simce afectan la tendencia al cambio. Si bien los modelos del año 2010 podrían verse afectados por la omisión de los efectos fijos por año-que controlan por factores constantes en los establecimientos- parece poco probable que factores constantes en el tiempo se correlacionen con los cambios en las características de los establecimientos incluidas en los modelos y, por ende, parece poco probable que se haya generado mayor sesgo en los modelos de dicho año.

Considerando lo anterior, este estudio no permite concluir respecto de cuál es la magnitud del efecto del cambio de las características de los estudiantes en el porcentaje de estudiantes que se traslada para el periodo de estudio. Aun así, se puede afirmar la existencia de una relación entre los cambios en el valor absoluto del precio y la tendencia al cambio en la muestra, y entre los cambios en el valor absoluto del Simce y la tendencia al cambio en la población. Que existan tales asociaciones, confirmaría que los cambios en algunas características de los establecimientos influyen en la tendencia al cambio de escuela, pese a que no se puede asegurar qué tanto del efecto estimado se debe a una variación de precio/Simce, y cuánto a variaciones en otras características de los establecimientos asociadas con variaciones de precio/Simce. A pesar de ello, los modelos de 2010 en específico mostrarían evidencia de que las modificaciones en el precio y del Simce influirían efectivamente en la tendencia al cambio.

\section{Conclusiones}

Los análisis realizados permiten concluir que hay cambios en las características de las escuelas que afectan el cambio de establecimiento. 
Si bien al pasar de ser un establecimiento de un solo género a uno mixto (o viceversa) no tiene un efecto, y no se puede afirmar que los cambios en los precios tengan efectos para el conjunto de establecimientos de niños y jóvenes en Chile (pero sí para los establecimientos de la muestra); incrementos en el valor absoluto del cambio en Simce sí se asociarían a aumentos en el porcentaje de estudiantes que se cambian de establecimiento. Adicionalmente, en el año 2010, aumentos en la satisfacción de los padres con la calidad del establecimiento propiciaría una menor tendencia al cambio de escuela en educación básica. Esto respaldaría los planteamientos de los teóricos del CS, de que las familias tienen preferencias por las características de los establecimientos, de modo que cuando un establecimiento las modifica, cambia la tendencia al cambio de escuela.

El efecto de los cambios en las características de los establecimientos en la tendencia al cambio de escuela no siempre es el mismo. La asociación entre el valor absoluto del cambio en Simce y la tendencia al cambio de escuela es mayor en la enseñanza media y entre el valor absoluto del cambio en precio y la tendencia al cambio de escuela es menor en este nivel que en educación básica. Esto sugiere que la valoración de los padres por distintos aspectos de los establecimientos es diferente según la etapa en la que el estudiante se encuentra.

Probablemente, y como lo sugieren los modelos de 2010, además del Simce y del precio existen otros aspectos de los establecimientos que inciden en el ajuste estudiante-escuela. Futuras investigaciones podrían ahondar en ellos y en cómo sus cambios afectan la movilidad escolar.

Por otro lado, la evidencia encontrada no permite respaldar que, como planteaban los teóricos del CS, mayor competencia comunal promueva mayor cambio de escuela, en tanto el cambio de escuela no se ve afectado por alternaciones en la competencia del mercado local. Nuevos desarrollos investigativos habrán de ahondar en si la competencia afecta la elección de escuela, o la voz de los padres en los establecimientos, promoviendo por otra vía, la prometida mejora en la calidad. 
Los resultados aquí encontrados son un primer indicio sobre los efectos de las preferencias y de la competencia en el cambio escolar, sin embargo, existen limitaciones en la definición de mercado relevante aquí utilizada. Es evidente que hay familias que envían a sus hijos a establecimientos de comunas distintas a la suya (Donoso \& Arias, 2013), pero que también los estudiantes tienden a recorrer distancias pequeñas (Canals et al., 2015). De este modo, la inclusión de coordenadas geográficas de los estudiantes en futuros estudios contribuirá a desarrollar nuevas estimaciones del efecto de la competencia en el cambio de escuela, con delimitaciones de los mercados más adecuadas a la realidad.

\section{Referencias}

Alderighi, M. \& Piga, C. A. (2012). Localized competition, heterogeneous firms and vertical relations. The journal of industrial economics, 1(60), 46-74. http://dx.doi.org/10.1111/j.1467-6451.2012.00472.x

Altonji, J., Huan, C.-I., \& Taber, C. (2010). Estimating the cream skimming effect of school choice. (Working Paper Series, 16.579). http://dx.doi. org/10.3386/w16579

Álvarez, A. (2001). Del Estado docente a la sociedad educadora: ¿un cambio de época? Revista Iberoaméricana de Educación, 26. Recuperado de http:// rieoei.org/rie26a02.htm

Ansión, J., Lazarte, A., Matos, S., Rodríguez, J., y Vega-Centeno, P. (1998). Educación: la mejor herencia. Lima: Pontificia Universidad Católica del Perú.

Arum, R. (1996). Do private schools force public schools to compete? American Sociological Review, 1(61), 29-46. http://dx.doi.org/10.2307/2096405

Association of London Goverment (2005). Breaking point. Examining the disruption caused by pupil mobility. ALG Mobility Report. London: autor.

Barrow, L. \& Rouse, C. E. (2004). Using market valuation to assess public school spending. Journal of Public Economics, 88, 1747-1769. http:// dx.doi.org/10.3386/w9054

Bell, C. (2005). All choices created equal? How good parents choose "failling" schools? (Working Paper). New York: National Center for the Study of Privatization in Education, Columbia University.

Bernal, J. (1999). Elección de escuela, clase social y fuerzas del mercado. Recuperado de http://webcasus.usal.es/orgyprof/_private/Lecturas/ Choiceschool.pdf 
Borland, M. V. \& Howsen, R. M. (1993). Students' academic achievement and the degree of market concentration in education. Economics of Education Review, 1(11), 31-39. http://dx.doi.org/10.1016/02727757(92)90019-y

Bowblis, J. R. \& North, P. (2011). Geographic market definition: The case of medicare-reimbursed skilled nursing facility care. Inquiry, 2(48), 138-154. http://dx.doi.org/10.5034/inquiryjrnl_48.02.03

Canales, M., Bellei, C., y Orellana, V. (2016, en prensa). ¿Por qué elegir una escuela particular subvencionada? Sectores medios emergentes y elección de escuela en un sistema de mercado. Revista Estudios Pedagógicos.

Canals, C. (2013). Factores sociales y geográficos que inciden en la elección de escuela (Tesis de grado inédita). Universidad de Chile, Santiago, Chile.

Canals, C., Meneses, F., Serra, C., y Ministerio de Educación, Mineduc. (2015). Aspectos geográficos de los cambios de estudiantes entre establecimientos escolares. Estudios de Política Educativa, 1(1), 180-202. Recuperado de http://centroestudios.mineduc.cl/tp_enlaces/portales/ tp5996f8b7cm96/uploadImg/File/Revista/Revista_Estudios_Politica_ Educativa_PDFFINAL.pdf

Chernew, M., Gowrisankaran, G., \& Fendrick, A. M. (2002). Payer type and the returns to bypass surgery: Evidence from hospital entry behavior. Journal of health economics, 21(3), 451-474. http://dx.doi.org/10.3386/ w8632

Chubb, J. E. \& Moe, T. M. (1990). Politics markets and America's schools. Washington, D.C.: The Brookings Institution.

Cohen-Zada, D. (2009). An alternative instrument for private school competition. Economics of Education review, 1(28), 29-37. http://dx.doi. org/10.1016/j.econedurev.2007.11.001

Córdoba, C. (2006). Elección de escuela en sectores pobres de Chile. Vincent Dupriez. Trabajo presentado en el simposio Políticas educacionales y gestión de sistemas escolares - PENS/EDU - 1. Departamento de Sociología VI. Facultad de Educación. Universidad Complutense, Madrid.

Cornejo, R. (2006). El experimento educativo chileno 20 años después: una mirada crítica a los logros y falencias del sistema escolar. Revista Iberoaméricana sobre Calidad, Eficacia y Cambio en Educación (REICE), 1(4), 118-129.

Corvalán, J. y Román, M. (2012). La permanencia de escuelas de bajo rendimiento crónico en el cuasi mercado educativo chileno. Revista Uruguaya de Ciencia Política, 21(1), 43-65. 
Cotterill, R. W. (1986). Market power in the retail food industry: Evidence from Vermont. The Review of Economics and Statistic, 3(68), 379-386. http://dx.doi.org/10.2307/1926014

Dauter, L. \& Fuller, B. A. (2011). How diverse schools affect student mobility: Charter, magnet, and newly built Institutions in Los Angeles (Working paper). Berkeley, CA: Policy Analysis for California Education.

De la Cruz, P. (2006). La educación formal en Chile desde 1973 a 1990: un instrumento para el proyecto de Nación. Recuperado de https://halshs. archives-ouvertes.fr/halshs-00104282/document

Del Cueto, C. (2004). Estrategias educativas de las clases medias en urbanizaciones cerradas del gran Buenos Aires. Espiral, 31(11), 249276.

Dijkgraaf, E., Van Der Geest, S., Gradus, R., \& De Jong, M. (2008). School choice and competition: Evidence from The Netherlands (Discussion paper). Amsterdam: Tinbergen Institute.

Donoso, S. \& Arias, O. (2013). Desplazamiento cotidiano de estudiantes entre comunas de Chile. Eure, 39 (116), pp.39-73.

Eadie, S., Eisner, R., Miller, B., \& Wolf, L. (2013). Student mobility patterns and achievement in Wisconsin. Recuperado de https://www.lafollette.wisc. edu/images/publications/workshops/2013-DPI_mobility.pdf

Efrón, B. (1992). Missing data, imputation and the bootstrap (Technical Report $\left.\mathrm{n}^{\circ} 153\right)$. Stanford, CA: Stanford University, Division of Biostatistics.

Elacqua, G. y Fábrega, R. (2004). El consumidor de la educación: el actor olvidado de la libre elección de escuelas en Chile. Recuperado de http://www.uai. cl/images/sitio/docentes/documentos/consumidor_educacion.pdf

Elacqua, G., Santos, H., Urbina, D. y Martínez, M. (2001). ¿Estamos preparados para cerrar las malas escuelas en Chile? Impacto sobre equidad en el acceso a educación de calidad. Proyecto Fonide F511083. Santiago de Chile: Ministerio de Educación.

Elacqua, G., Martínez, M., Santos, H., \& Urbina, D. (2012). School closures in Chile: Access to quality alternatives in a school choice system. Estudios de Economía, 2(39), 179-202. http://dx.doi.org/10.4067/ s0718-52862012000200005

Elzinga, K. G. (1981). Defining geographic market boundaries. Antitrust Bull, 26, 739-752.

Elzinga, K. G. \& Hogarty, T. F. (1978). The problem of geographic market delineation revisited: The case of coal. Antitrust Bull, 23(1), pp.1-18. 
Fábrega, F., Aguirre, C., Blanco, C., Canals, C., Mena, C., y Paulus, N. (2013). Diseccionando el "voucher" escolar. Trabajo presentado en el XXIX Congreso ALAS, Asociación Latinoamericana de Sociología, Santiago de Chile.

Friedman, M. (1955). The role of government in education in economics and the public interest. New Brunswick, NJ: Rutgers University Press.

Friedman, M. \& Friedman, R. (1982). Capitalism and freedom. Chicago: The University of Chicago Press.

Friedman, M. (1995). Public schools: Make them private (Cato institute briefing paper n²3) Recuperado http://www.cato.org/sites/cato.org/files/pubs/ pdf/bp023.pdf

Gallego, F. y Sapelli, C. (2007). El financiamiento de la educación en Chile: una evaluación. Revista Pensamiento Educativo, 1(40), 263-284.

Gallego, F. \& Hernando, A. (2009). School choice in Chile: Looking at the demand size (Documento de trabajo, IE-PUC, n 356). http://dx.doi. org/10.2139/ssrn.1725911

Geller, C. R., Sjoquist, D. L., \& Walker, M. B. (2006). The effect of private school competition on public school performance in Georgia. Public Finance Review, 1(34), 4-32. http://dx.doi.org/10.1177/1091142105283631

Gómez, D., Chumacero, R., \& Paredes, R. (2012). School choice and information. Estudios de Economía, 2(39), 143-157.

Hanuschek, E., Kain F., \& Rivkin, S. (2001). Disruption versus Tiebout improvement: The costs and benefits of switching schools. Recuperado de http://hanushek.stanford.edu/sites/default/files/publications/ Hanushek\%2BKain\%2BRivkin\%202004\%20JPubE\%2088(9).pdf

Hirschman, A. O. (1970). Exit, voice, and loyalty. Responses to decline in firms, organizations, and states. Cambridge: Harvard University Press.

Hsieh, C.-T. \& Urquiola, M. (2003). When schools compete, how do they compete? An assesment of ChileOs nationwide school voucher program (NBER Working Paper No. 10008). http://dx.doi.org/10.3386/w10008

Jepsen, C. (2002). The role of aggregation in estimating the effects of private school competition on student achievement. Journal of Urban Economics, 3(52), 477-500. http://dx.doi.org/10.1016/s0094-1190(02)00530-2

Joiko, S. (2012). El cuasi-mercado educativo en Chile: desarrollo y consecuencias. Diálogos Educativos, 23(12), 148-174. Recuperado de http://www.dialogoseducativos.cl/revistas/n23/joiko/ 
Jofré, G. (1998). El sistema de subvenciones en educación: la experiencia chilena. Estudios Públicos, 32, 193-237. Recuperado de http://opech.cl/ bibliografico/Doc_Financiamiento/sistema_subvenciones_educacion_ chilena.pdf

Loeb, S. \& Valant, J. (2011). Student mobility in Milwaukee: The effect of school transfers on mobile and non-mobile students. Trabajo presentado en The Association for Education Finance and Policy Annual Conference. Seattle, WA. Recuperado de https://cepa.stanford.edu/sites/default/files/ Loeb_Valant_MobilityinMilwaukee_AEFP2011.pdf

Machin, S., Telhaj, S., \& Wilson, J. (2006). The mobility of english school children. Fiscal Studies, 3(27), 253-280. http://dx.doi.org/10.1111/ j.1467-8578.2006.00035.x

Madero, C. (2012). Educación escolar católica en Chile, ¿quién elige a quien? Un análisis de la elección de escuela y la selección de estudiantes en el sistema educativo chileno. Trabajo presentado en el Tercer Encuentro Sociedad Chilena de Políticas Públicas. Santiago de Chile.

Makovec, M., Mizala, A., \& Barrera, A. (2010). Parental decisions in a choice based school system: Analyzing the transition between primary and secondary school (Documento de trabajo, Serie Economía n 269). Santiago de Chile: Centro de Economía Aplicada, Universidad de Chile.

McMillan, R. (2000). Competition, parental involvement, and public school performance. En National Tax Association, Proceedings from the annual meeting of the National Tax Association (pp. 150-155). Washington, DC: autor.

Mizala, A. y Romaguera, P. (1998). Desempeño escolar y elección de establecimientos: la experiencia chilena (Documento de trabajo, Serie Economía, no 36). Santiago de Chile: Centro de Economía Aplicada, Universidad de Chile.

Morrisey, M., Sloan, F., \& Valvona, J. (1988). Defining geographic markets for hospital care. Law and Contemporary Problems, 2(51), 165-194. http://dx.doi.org/10.2307/1191730

Ortiz, I. (2012). En torno a la validez del Sistema de Medición de la Calidad de la Educación en Chile. Estudios Pedagógicos, 38(2), 355-373.

Pamies, J., Carrasco, S. Beremenyi, B. A., y Casalta, V. (2011). ¿Matrícula viva o movilidad del alumnado? Resituando las estrategias de incorporación escolar en Barcelona en el marco de globalización. En F. J. García Castaño y N. Kressova. (Coords.), Actas del I Congreso Internacional sobre Migraciones en Andalucía (pp. 413-420). Granada: Instituto de Migraciones. 
Paredes, R., Chumacero, R., \& Gallegos, J. (2012). School choice and switching costs. Recuperado de http://www.faceauv.cl/cladea2015/images/stories/ track2/CLADEA_2015_submission_299_2_.pdf

Raczynski, D. y Hernández, M. (2011). Elección de establecimiento, imágenes, valoraciones y conductas de las familias y segregacion social escolar. Santiago de Chile: Asesorías para el desarrollo.

Robinson, J. C. \& Luft, H. (1985). The impact of hospital market structure on patient volumen, average length of stay, and the cost of care. Journal of Health Economics, 4(4), 333-356. http://dx.doi.org/10.1016/01676296(85)90012-8

Shrieves, R. E. (1978). Geographic market areas and market structure in the bituminous coal industry. Antitrust Bull, 23, 589-625.

Usón, V. (1993). Karl Manheim (1893-1947): La construcción social de la libertad. REIS, 62, 83-98. http://dx.doi.org/10.2307/40183638

Van Zantes, A. (2007). Reflexividad y elección de la escuela por los padres de la clase media en Francia. Revista de Antropología Social, 16, 245-278.

Weis, J. (2002). Market power and power markets . Interfaces, 32(5), 37-46.

Zamora, G. (2011). Movilidad escolar en Chile. Análisis de las implicancias para la calidad y equidad de la educación. Estudios Pedagógicos, 1(37), 53-69.

Zamora, G. y Moforte, C. (2013). ¿Por qué los estudiantes se cambian de escuela? Análisis desde las decisiones familiares. Perfiles Educativos, 35(140), 48-62. http://dx.doi.org/10.1016/s0185-2698(13)71821-x

Recibido: 30/04/2015

Aceptado: 19/07/2016 